\title{
Correction to: Fabrication of Enzymatic Biofuel Cell with Electrodes on Both Sides of Microfluidic Channel
}

\author{
Haroon Khan ${ }^{1}$. Chul Min Kim ${ }^{1}$ - Sung Yeol Kim ${ }^{1}$ - Sanket Goel ${ }^{2}$. Prabhat K. Dwivedi ${ }^{3}$. Ashutosh Sharma ${ }^{3,4}$. \\ Young Ho Kim ${ }^{5}$. Gyu Man Kim ${ }^{1}$
}

Published online: 24 May 2019

(c) Korean Society for Precision Engineering 2019

\section{Correction to: \\ International Journal of Precision Engineering and Manufacturing-Green Technology https://doi.org/10.1007/s40684-019-00056-x}

The original version of this article unfortunately contains a mistake. The acknowledgement section of the above article is incorrect. The correct acknowledgements are as follows:

Acknowledgements This work was supported by the National Research Foundation of Korea Grant (NRF-2017R1A2B4004966 and NRF-2012K1A3A1A19038448) funded by the Korean Government (KMEST), and "Project of Advanced Technology Center (10077414, 2017)" funded by the Korean Government (MOTIE).
Publisher's Note Springer Nature remains neutral with regard to jurisdictional claims in published maps and institutional affiliations.
The original article can be found online at https://doi.org/10.1007/ s40684-019-00056-x.

Gyu Man Kim

gyuman.kim@knu.ac.kr

Haroon Khan

mechy_365@yahoo.com

Chul Min Kim

faithfulsaint@hanmail.net

Sung Yeol Kim

sykimknu@knu.ac.kr

Sanket Goel

sgoel@hyderabad.bits-pilani.ac.in

Prabhat K. Dwivedi

prabhatd@iitk.ac.in

Ashutosh Sharma

ashutos@iitk.ac.in
Young Ho Kim

yhkim@dgmif.re.kr

1 School of Mechanical Engineering, Kyungpook National University, Daegu 41566, South Korea

2 Birla Institute of Technology and Science, Pilani, Rajasthan 333031, India

3 Centre for Nanosciences, Indian Institute of Technology Kanpur, Kanpur 208016, India

4 Department of Chemical Engineering, Indian Institute of Technology, Kanpur 208016, India

5 Medical Device Development Centre, Daegu-Gyeongbuk Medical Innovation Foundation, Daegu 41061, South Korea 